\title{
Accelerated Bioorthogonal Conjugation: A Practical Method for the Ligation of Diverse Functional Molecules to a Polyvalent Virus Scaffold
}

\author{
Sayam Sen Gupta, ${ }^{1}$ Jane Kuzelka, ${ }^{1}$ Pratik Singh, ${ }^{2}$ Warren G. Lewis, ${ }^{1}$ Marianne Manchester, ${ }^{2}$ and M. G. Finn*,1
}

\section{Supporting Information}

General Characterization. All CPMV conjugates were characterized by analytical size exclusion FPLC. The representative trace shown in Figure S1 is of CPMV-5; other conjugates show chromatograms that are essentially identical, unless indicated otherwise. Note the trace monitored at $496 \mathrm{~nm}$, showing fluorescein covalently bound to CPMV. Substrate loadings were calculated using the $496 \mathrm{~nm}$ absorbance values. SDS-PAGE analysis of all conjugates was also performed. Gels essentially identical to that shown in Figure 2 (lane 2) were obtained for all samples, unless indicated otherwise. The EMAN program was used to measure particle diameter (www.software-ncmi.bcm.tmc.edu/ncmi/homes/stevel/EMAN/doc).

CPMV-8b. The attachment of $\mathbf{8 b}$ to CPMV was further confirmed by monitoring the rate of aggregation of CPMV-8b with the dimeric Galectin-4 at $490 \mathrm{~nm}$, where no absorbance of either icosahedral CPMV-8b or galectin-4 is observed (Figure S2). Gel formation was rapid at virus concentrations of $1.0 \mathrm{mg} / \mathrm{mL}$.

CPMV-13. Analytical size exclusion FPLC of CPMV-13 is shown in Figure S3. The more rapid elution of CPMV-PEG relative to wild-type CPMV indicates a substantial size increase of the particle.

CPMV-14. Western blots of conjugate CPMV-14 using antibodies against both CPMV and human Tfn show that high molecular weight bands react with both antibodies, indicating modification of CPMV with Tfn (Figure S4).

Figure S1. Size-exclusion FPLC traces of CPMV-5. Traces were monitored at 3 different wavelengths.

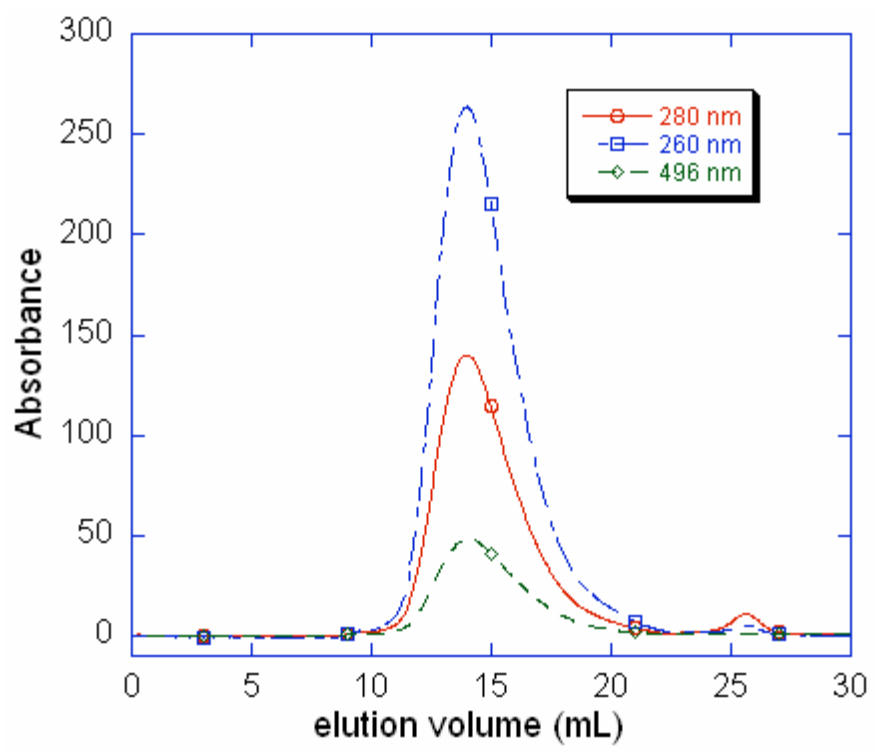


Figure S2. Time course of agglutination monitored at $490 \mathrm{~nm}$ for a mixture of galectin-4 $(300 \mu \mathrm{g} / \mathrm{mL}, 50 \mu \mathrm{L}$ of $)$ and CPMV-8b $(1.0 \mathrm{mg} / \mathrm{mL}, 77 \mu \mathrm{L})$ in phosphate-buffered saline.

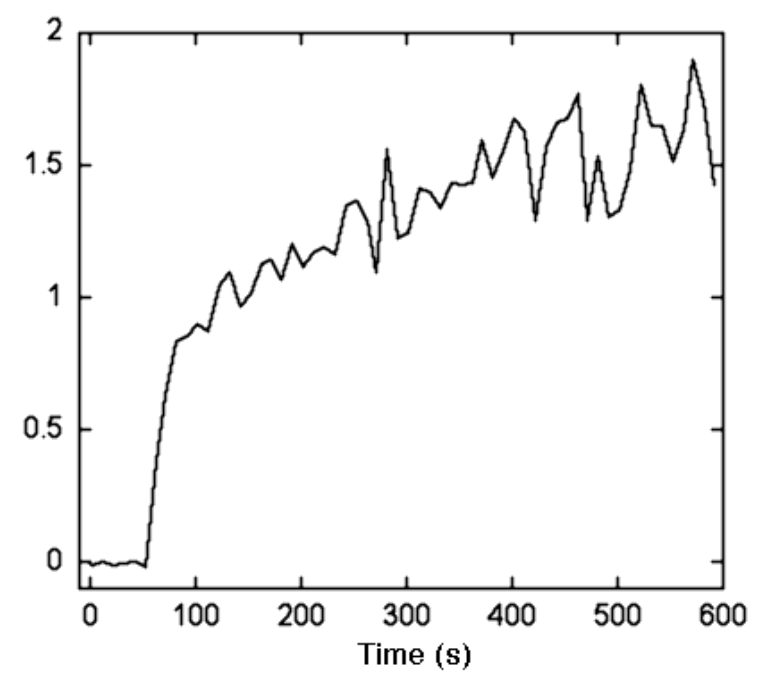

Figure S3. Size-exclusion FPLC of wild-type CPMV and CPMV-13. Protein from disassembled particles would appear at retention times greater than that of the observed peaks. Both samples display $\mathrm{A}_{260} / \mathrm{A}_{280}$ ratios that are characteristic of intact, RNA-containing capsids. The void volume of the column is $10 \mathrm{~mL}$.

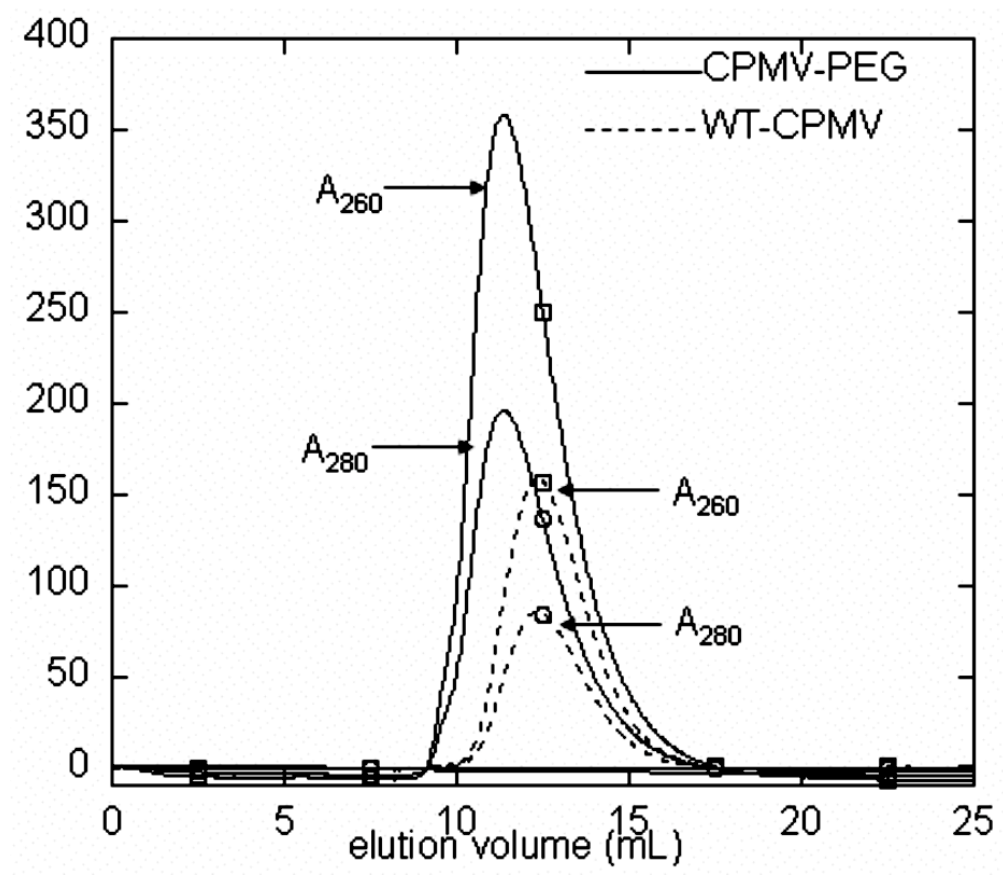


Figure S4. Western blots of CPMV-14 using polyclonal antibodies against CPMV or Tfn. Proteins denatured on a $4-12 \%$ bis-tris gel were transferred to a PVDF membrane and blocked with $5 \%$ milk. The membrane was then incubated with antibodies against CPMV (produced by the Manchester laboratory, 1:2000 dilution) or human Tfn (goat, Sigma; 1:2000 dilution). Subsequent incubation of HRP conjugates of goat-anti-rabbit (for antiCPMV) or rabbit-anti-goat (for anti-Tfn), used at the manufacturer's recommended dilution followed by TMB membrane peroxidase substrate permitted visualization of the protein bands. Samples are as follows: 4 (lanes 1, 5); 14 (lanes 2, 6); molecular weight marker (lanes 3, 7); CPMV-(14) (lanes 4, 8).

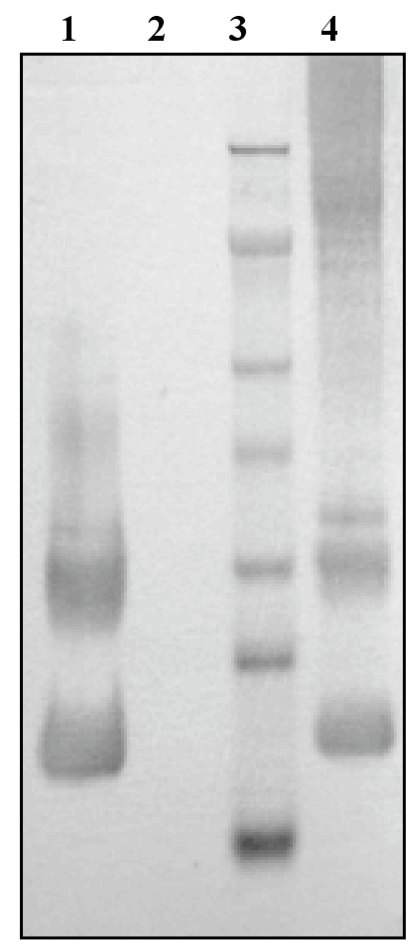

Anti-CPMV

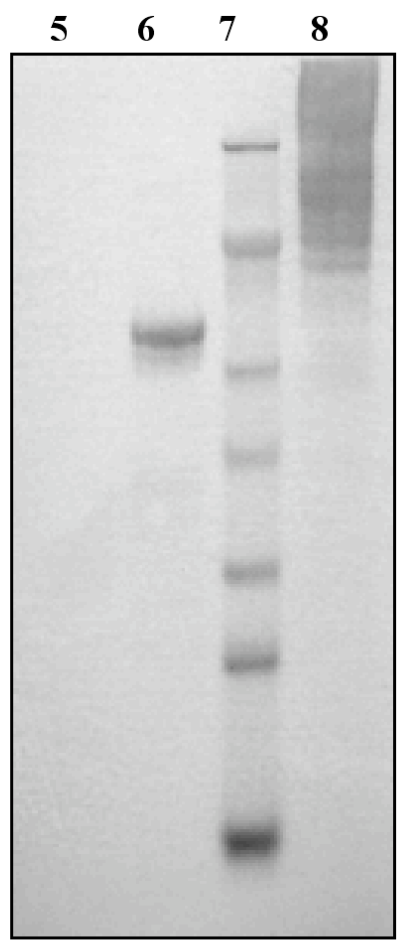

Anti-Tfn 